\title{
Probabilidade de hipertensão arterial a partir de indicadores antropométricos em adultos
}

\author{
Probability of arterial hypertension from \\ anthropometric measures in adults
}

Anderson Zampier Ulbrich ${ }^{1,2}$, Renata L. Bertin ${ }^{3}$, Rodrigo Bozza ${ }^{4}$, Antônio Stabelini Neto', Gustavo Zampier dos Santos Lima ${ }^{5}$, Tales de Carvalho', Wagner de Campos ${ }^{4}$

\section{RESUMO}

Objetivo: Associar medidas antropométricas com hipertensão arterial e desenvolver modelo matemático para estimar essa probabilidade. Sujeitos e métodos: Estudo transversal com 3.445 adultos de ambos os sexos entre 18 e 60 anos. Avaliaram-se o índice de massa corporal $(\mathrm{IMC})$, razão cintura estatura (RCE), perímetro da cintura ( $P C)$, além da idade e sexo. A pressão arterial foi avaliada e classificada segundo recomendações da Organização Mundial da Saúde (OMS) e da Sociedade Brasileira de Hipertensão (SBH). Utilizaram-se análise de frequência de casos, correlação e regressão logística entre as variáveis, com $p<0,05$. Resultados: Os homens apresentaram duas vezes mais chances de hipertensão que as mulheres, e, para cada ano de vida, essa chance aumenta em 1,04 vez. As chances de hipertensão dos considerados com sobrepeso foram de quase duas vezes e, nos obesos, quadriplicavam. Conclusão: Alterações da composição corporal com o avanço da idade, principalmente nos homens, associaram-se com hipertensão, sendo sua avaliação relevante na identificação dessa doença. Arq Bras Endocrinol Metab. 2012;56(6):351-7

\section{Descritores}

Antropometria; hipertensão; fatores de risco

\begin{abstract}
Objective: To associate anthropometric measures with arterial hypertension and develop a mathematical model to estimate this probability. Subjects and methods: A cross-sectional study was carried out with 3,445 adults of both sexes, between 18 and 60 years of age. Body mass index (BMI), waist-to-height ratio (WHR), waist circumference $(\mathrm{HC})$, in addition age and sex were assessed. Blood pressure (BP) was measured and classified according to World Health Organization (WHO) and Brazilian Society of Hypertension (SBH) recommendations, using frequency analyses, correlation and logistic regression between variables, with $p<0.05$. Results: Males were twice as likely as woman to have hypertension, and for each year of life, the chance of hypertension increased 1.04 times. Odds ratio of hypertension in overweight individual increased two times, and in obese subjects, four times. Conclusion: Changes in body composition with advancing age, especially in men, were associated with hypertension, and assessment of these changes was relevant in the identification of this disease. Arq Bras Endocrinol Metab. 2012;56(6):351-7
\end{abstract}

\section{Keywords}

Anthropometry; hypertension; risk factors

Centro de Ciências da Saúde e do Esporte (Cefid), Programa de Pós-Graduação em Ciência do Movimento Humano, Universidade do Estado de Santa Catarina (Udesc), Florianópolis, SC, Brasil ${ }^{2}$ Coordenação de Aperfeiçoamento de Pessoal de Nível Superior (Capes), Brasília, DF, Brasil ${ }^{3}$ Departamento de Nutrição, Universidade Regional de Blumenau (Furb), Blumenau, SC, Brasil ${ }^{4}$ Departamento de Educação Física, Programa de PósGraduação em Educação Física, Universidade do Federal do Paraná (UFPR), Curitiba, PR, Brasil ${ }^{5}$ Escola de Ciências e Tecnologia, Universidade Federal do Rio Grande do Norte (ECT/ UFRN), Natal, RN, Brasil

Correspondência para: Anderson Zampier Ulbrich Centro de Ciências da Saúde e do Esporte

Rua Pascoal Simone, 358 88080-350 - Florianópolis, SC, Brasil anderson_u@hotmail.com

Recebido em 19/Jul/2011 Aceito em 26/Jul/2012 


\section{INTRODUÇÃO}

A tualmente, a hipertensão arterial sistêmica (HAS) Atem sido considerada problema de saúde pública de etiologia multifatorial, descrita como um dos maiores fatores de risco prevalentes para desenvolvimento de doenças cardiovasculares, cerebrovasculares e insuficiência cardíaca congestiva. Esses agravos são importantes causas de morbimortalidade e apresentam elevado custo social (1-4).

A HAS tem sido responsável, em média, por 30\% das mortes em indivíduos adultos no mundo (4). No Brasil, a Sociedade Brasileira de Hipertensão (5) relatou prevalência de HAS na população urbana adulta entre $22,3 \%$ e $43,9 \%$ e destacou pesquisas apontando a grande ascendência de HAS entre sujeitos acima do peso, para ambos os sexos. Recentemente, Chirinos e cols. (6), por meio de dados do National Health and Nutrition Examination Survey (NHANES) de 1999-2004, demonstraram ser o índice de massa corporal (IMC) um significante preditor para ocorrência da HAS, com razão de chances de 1,04 para cada cinco unidades do IMC e associado a outros fatores como perímetro da cintura, razão cintura estatura, idade e sexo.

Com esse enfoque, o presente estudo objetivou verificar a prevalência de hipertensão arterial assim como associar o diagnóstico de hipertensão arterial com medidas antropométricas a ponto de verificar o modelo matemático desse acometimento. Além disso, esse estudo buscou analisar, a partir de um modelo matemático, uma previsão que estime a probabilidade de hipertensão arterial e qual a influência dos dados antropométricos com a hipertensão.

\section{MÉTODO}

\section{População}

Estudo de base populacional, de caráter transversal, foi realizado com 3.445 adultos, sendo 2.156 mulheres e 1.289 homens, entre 18 e 60 anos de idade, residentes na zona urbana da cidade de Curitiba, Paraná, Brasil. A amostra foi obtida do banco de dados dos projetos desenvolvidos pela Secretaria de Esporte e Lazer e Paraná Esporte.

Todos os procedimentos da coleta dos dados do presente estudo foram aprovados pelo Comitê de Ética de Pesquisa em seres humanos do Setor de Ciências da Saúde da Universidade Federal do Paraná (UFPR).

\section{Medidas antropométricas}

As medidas antropométricas (massa corporal e estatura) foram coletadas por professores de Educação Física treinados. As medições foram todas realizadas em duplicata, sendo uma terceira medida realizada quando os dois primeiros valores apresentavam diferenças superiores ou iguais a $0,2 \mathrm{~kg}$ para massa corporal e estatura e $0,2 \mathrm{~cm}$ para estatura. A medida da massa corporal foi efetuada por balança digital, portátil, com capacidade máxima de $150 \mathrm{~kg}$ e escala de $100 \mathrm{~g}$, com o indivíduo descalço e usando roupas leves. A estatura foi aferida por meio do estadiômetro portátil, graduado em milímetros. As padronizações e aferições dessas medidas antropométricas foram todas desempenhadas pelas recomendações de Lohman e cols. (7).

Por meio da razão da massa corporal pela estatura ao quadrado dos indivíduos, foi calculado o IMC. Os valores do IMC foram categorizados por meio do estado nutricional, segundo a proposta da Organização Mundial da Saúde (8), seguindo os pontos de corte: normal: entre 18,5 a $24,99 \mathrm{~kg} / \mathrm{m}^{2}$; sobrepeso: 25 a $29,99 \mathrm{~kg} / \mathrm{m}^{2}$; obesidade: acima de $30 \mathrm{~kg} / \mathrm{m}^{2}$.

$\mathrm{O}$ perímetro da cintura (PC) foi mensurado no ponto médio entre o último arco costal e a crista ilíaca, utilizando-se uma fita antropométrica flexível, com escala de 0,1 centímetro e comprimento máximo de $150 \mathrm{~cm}$ (9). Os indivíduos foram avaliados em pé de frente para $\mathrm{o}$ avaliador, com as pernas unidas e os braços cruzados sobre o peito, e no momento da medida foi solicitado ao indivíduo que deixasse a musculatura abdominal relaxada. Os pontos de corte adotados foram de 88 e $102 \mathrm{~cm}$, para mulheres e homens, respectivamente (8).

Com essas medidas, foi realizado o cálculo da relação cintura estatura (RCE), usando como ponto de corte para risco coronariano valores acima de 0,5 (10).

\section{Pressão arterial}

A medida da pressão arterial sistólica (PAS) e diastólica (PAD) foi realizada e classificada segundo os pressupostos estabelecidos nas "VI" Diretrizes Brasileiras de Hipertensão (5). Todas as medidas da pressão arterial foram realizadas com o indivíduo na posição sentada, com o braço direito apoiado na altura da região do co- $^{-}$ ração após permanecer cinco minutos na posição sentada, as pernas descruzadas, não ter se exercitado fisicamente 60 a 90 minutos antes, pelo menos 30 minutos sem ingestão de cafeína, bebidas alcoólicas e consumo de cigarros (5). Aferiram-se a PAS e a PAD por meio 
dos equipamentos de esfigmomanômetro aneroide e estetoscópio. Foi considerado PAS, fase I, o início dos ruídos auscultatórios e, com a extinção dos ruídos auscultatórios, considerou-se como PAD (5).

A pressão arterial foi classificada de três formas diferentes para a análise posterior. $\mathrm{Na}$ primeira, foi diagnosticada a hipertensão a partir de valores sistóli$\cos (\mathrm{PAS}) \geq 140 \mathrm{mmHg}$. Na segunda, a hipertensão foi classificada a partir de valores da pressão diastólica (PAD) acima de $85 \mathrm{mmHg}$. Dessa forma, os indivíduos foram categorizados em normais ou hipertensos. A terceira categorizou os indivíduos em três classes: normal, pré-hipertensão e hipertensão. Os pontos de corte da PAS e PAD foram pré-estipulados como: normal (PAS $<130 \mathrm{mmHg}$ e PAD < $85 \mathrm{mmHg}$ ); pré-hipertensão (PAS $\geq 130$ a $<140 \mathrm{mmHg}$ e/ou PAD $\geq 85$ a $\leq 89$ $\mathrm{mmHg}$ ); hipertensão (PAS $\geq 140 \mathrm{mmHg}$ e/ou PAD $\geq$ $90 \mathrm{mmHg})(5)$.

\section{Análise estatística}

Os dados foram processados e analisados no programa Statistical Program Software System (SPSS) para Windows versão 17,0 , e o nível de significância adotado foi de $5 \%$.

Foi verificada a porcentagem de sujeitos para as categorias de idade, sexo, antropométricas e de pressão arterial. A correlação de Pearson foi dada entre as variáveis independentes (idade, sexo, IMC, RCE e PC).

A criação do modelo estatístico foi realizada por meio da regressão logística com método Backward for Wald, que parte de um modelo inicial que contém todas as variáveis independentes escolhidas para entrar (Tabela 1) na análise e a partir dele exclui-se uma variável independente de cada vez de acordo com o critério que determina qual variável apresenta pouca significância (ficar acima de 0,05 no p-valor) na variável de resposta.

O modelo final foi construído com a categorização dos indivíduos tanto para a PAS quanto para PAD normal e hipertenso, sendo observada a avaliação sobre o desempenho geral, ou seja, a variação ocorrida no log da razão de chances do conjunto de variáveis independentes do modelo pelo valores do Cox \& Snell R square (R-ajustado). Por meio da categorização da hipertensão em três categorias, foi utilizada a regressão logística multinomial. Neste contexto foi utilizado como variável dependente de referência a categoria normal de pressão, associada com as demais (pré-hipertensivo e hipertensivo) por meio das variáveis independentes: idade, sexo, IMC, RCE e PC.

\section{RESULTADOS}

A distribuição percentílica de casos das diferentes variáveis analisadas neste estudo pode ser vista na tabela 1 , havendo boa representatividade entre as faixas etárias, para o sexo feminino e as variáveis independentes do estudo.

Tabela 1. Distribuição (n e \%) das variáveis do estudo entre homens, mulheres e no total

\begin{tabular}{|c|c|c|c|c|c|c|}
\hline & \multicolumn{2}{|c|}{ Masculino } & \multicolumn{2}{|c|}{ Feminino } & \multicolumn{2}{|c|}{ Total } \\
\hline & $\mathbf{N}$ & $\%$ & $\mathbf{N}$ & $\%$ & $\mathbf{N}$ & $\%$ \\
\hline \multicolumn{7}{|l|}{ Idade } \\
\hline $18-29$ & 365 & 28,3 & 627 & 29,1 & 992 & 28,8 \\
\hline $30-39$ & 326 & 25,3 & 527 & 24,4 & 853 & 24,8 \\
\hline $40-49$ & 366 & 28,4 & 586 & 27,2 & 952 & 27,6 \\
\hline $50-60$ & 232 & 18,0 & 416 & 19,3 & 648 & 18,8 \\
\hline \multicolumn{7}{|l|}{ PC } \\
\hline Normal & 898 & 69,7 & 1.220 & 56,6 & 2.118 & 61,5 \\
\hline Elevado & 391 & 30,3 & 936 & 43,4 & 1.327 & 38,5 \\
\hline \multicolumn{7}{|l|}{ RCE } \\
\hline Normal & 600 & 46,6 & 1.249 & 57,9 & 1.849 & 53,7 \\
\hline Elevado & 687 & 53,4 & 907 & 42,1 & 1.594 & 46,3 \\
\hline \multicolumn{7}{|l|}{ IMC } \\
\hline Normal & 563 & 43,7 & 1.309 & 60,7 & 1.872 & 54,4 \\
\hline Sobrepeso & 537 & 41,7 & 579 & 26,9 & 1.116 & 32,4 \\
\hline Obesidade & 188 & 14,6 & 268 & 12,4 & 456 & 13,2 \\
\hline \multicolumn{7}{|l|}{ PA } \\
\hline Normal & 876 & 68,0 & 1.788 & 82,9 & 2.664 & 77,3 \\
\hline Pré-hipertensão & 150 & 11,6 & 142 & 6,6 & 292 & 8,5 \\
\hline Hipertensão & 263 & 20,4 & 226 & 10,5 & 489 & 14,2 \\
\hline \multicolumn{7}{|l|}{ PAS } \\
\hline Normal & 1.132 & 87,8 & 2.029 & 94,1 & 3.161 & 88,3 \\
\hline Hipertenso & 157 & 12,2 & 127 & 5,9 & 284 & 8,2 \\
\hline \multicolumn{7}{|l|}{ PAD } \\
\hline Normal & 1.072 & 83,2 & 1.969 & 91,3 & 3.041 & 88,3 \\
\hline Hipertenso & 217 & 16,8 & 187 & 8,7 & 404 & 11,7 \\
\hline
\end{tabular}

IMC: índice de massa corporal; n: número de indivíduos; PAD: pressão arterial classificada pela diástole; PAS: pressão arterial classificada pela sístole; PA: pressão arterial classificada pela sístole ou diástole; $\mathrm{PC}=$ perímetro da cintura; $\mathrm{RCE}=$ razão cintura estatura.

Na tabela 2, estão apresentadas as razões de chances para ocorrência de hipertensão arterial diastólica a partir das variáveis antropométricas com valores acima dos pontos de corte propostos na literatura. Nos resul- 
tados, relata-se que a hipertensão diastólica associou-se com o sexo, a idade, o IMC e o PC. Os homens apresentaram 2,25 vezes mais chance de desenvolver hipertensão quando comparados com as mulheres, sendo ainda verificado que, para cada ano de vida, a chance de desenvolver essa doença aumenta em 1,04 vez. Com relação às medidas antropométricas, os considerados com sobrepeso (IMC $\geq 25 \mathrm{~kg} / \mathrm{m}^{2} \mathrm{e} \leq 30 \mathrm{~kg} / \mathrm{m}^{2}$ ) e obesos têm 1,66 e 3,63 mais chance, respectivamente, de apresentar hipertensão arterial diastólica que os sujeitos eutróficos. Por conseguinte, os sujeitos considerados com PC elevado têm 1,48 vez mais probabilidade de desenvolver a hipertensão que o grupo normal.

Tabela 2. Razão de chances de desenvolvimento de hipertensão arterial diastólica

\begin{tabular}{|c|c|c|}
\hline \multirow{2}{*}{ Variáveis } & Hipertensão & \multirow{2}{*}{ p-valor } \\
\hline & RC (IC95\%) & \\
\hline \multicolumn{3}{|l|}{ Sexo } \\
\hline Feminino & 1 & \\
\hline Masculino & $2,25(1,79-2,84)$ & $0,001^{\star *}$ \\
\hline \multicolumn{3}{|l|}{ Idade } \\
\hline & $1,04(1,02-1,05)$ & \\
\hline IMC & & $0,001^{* \star}$ \\
\hline Normal & 1 & \\
\hline Sobrepeso & $1,66(1,22-2,26)$ & $0,001^{\star \star}$ \\
\hline Obeso & $3,63(2,51-5,27)$ & \\
\hline \multicolumn{3}{|l|}{ PC } \\
\hline Normal & 1 & \\
\hline Elevado & $1,48(1,10-2,01)$ & $0,009^{\star \star}$ \\
\hline \multicolumn{3}{|l|}{ RCE } \\
\hline Normal & 1 & \\
\hline Elevado & $1,23(0,84-1,81)$ & 0,277 \\
\hline
\end{tabular}

RC: razão de chance; IC: intervalo de confiança; IMC: índice de massa corporal; PC: perímetro da cintura; RCE: razão cintura estatura. ${ }^{* *}$ Critério de associação e de permanência no modelo $=p<0,05$.

O modelo final para a probabilidade de desenvolver a hipertensão diastólica $\left(\mathrm{P}_{\mathrm{hd}}\right)$, a partir da análise de regressão apresentada na tabela 2 , ficou de acordo com a equação a seguir, sendo que o Cox \& Snell R square indica dessa forma que cerca de $84 \%$ das variações ocorridas são explicadas pelo conjunto de variáveis independentes (antropométricas).

$P_{\text {hd }}=\frac{e^{\left.-8,081+0,446^{*}(\text { sexo })+0,034^{*} \text { (idade }\right)+0,039 *(P C)+0,044^{*}(\mathrm{IMC})}}{1+\mathrm{e}^{\left.-8,081+0,446^{*}(\text { sexo })+0,034^{*} \text { (idade }\right)+0,039^{*}(\mathrm{PC})+0,044^{*}(\mathrm{IMC})}}$

Onde: sexo feminino $=0$; sexo masculino $=1$; idade: em anos; $\mathrm{PC}$ : valor absoluto em centímetros perímetro da cintura; IMC: valor absoluto do índice de massa corporal.
Na tabela 3, destacam-se as razões de chance de desenvolvimento da hipertensão arterial sistólica a partir do sexo, da idade e do IMC. Os homens têm 2,14 vezes mais chance de desenvolver hipertensão que as mulheres. O resultado da idade foi o mesmo que o da pressão arterial diastólica (aumento de 1,04 vez a cada ano). Quanto ao IMC, o grupo do sobrepeso tem 1,97 vez mais chance que o grupo normal, e o grupo dos obesos tem 4,15 vezes mais chance que o grupo normal.

Tabela 3. Razão de chances de desenvolvimento de hipertensão arterial sistólica

\begin{tabular}{|c|c|c|}
\hline \multirow{2}{*}{ Variáveis } & Hipertensão & \multirow{2}{*}{ p-valor } \\
\hline & RC (IC95\%) & \\
\hline \multicolumn{3}{|l|}{ Sexo } \\
\hline Feminino & 1 & \\
\hline Masculino & $2,14(1,66-2,77)$ & $0,001^{* *}$ \\
\hline \multicolumn{3}{|l|}{ Idade } \\
\hline & $1,04(1,03-1,06)$ & \\
\hline IMC & & $0,001^{* \star}$ \\
\hline Normal & 1 & \\
\hline Sobrepeso & $1,97(1,44-2,70)$ & $0,001^{\text {** }}$ \\
\hline Obeso & $4,15(2,95-5,54)$ & \\
\hline \multicolumn{3}{|l|}{ PC } \\
\hline Normal & 1 & \\
\hline Elevado & $1,33(0,94-1,89)$ & 0,98 \\
\hline \multicolumn{3}{|l|}{ RCE } \\
\hline Normal & 1 & \\
\hline Elevado & $1,17(0,75-1,83)$ & 0,47 \\
\hline
\end{tabular}

RC: razão de chance; IC: intervalo de confiança; IMC: índice de massa corporal; PC: perímetro da cintura; RCE: razão cintura estatura. ${ }^{* \star}$ Critério de associação e de permanência no modelo $=\mathrm{p}<0,05$.

O modelo final que calcula a probabilidade de desenvolver a hipertensão sistólica $\left(\mathrm{P}_{\mathrm{hs}}\right)$ ficou de acordo com a equação a seguir, sendo que o valor do Cox \& Snell R square demonstrou que cerca de $64 \%$ das variações ocorridas são explicadas pelo conjunto de variáveis independentes (antropométricas).

$P_{\text {hs }}=\frac{e^{-8,214+0,553^{*}(\text { sexo })+0,042^{*}(\text { idade })+0,03^{*}(\mathrm{PC})+0,046^{*}(\mathrm{IMC})}}{1+\mathrm{e}^{-8,214+0,553^{*}(\mathrm{sex} 0)+0,042^{*}(\text { idade })+0,03^{*}(\mathrm{PC})+0,046^{*}(\mathrm{IMC})}}$

Onde: sexo feminino $=0$; sexo masculino $=1$; idade: em anos; $P C$ : valor absoluto em centímetros perímetro da cintura; IMC: valor absoluto do índice de massa corporal.

Na tabela 4, são destacados os resultados da regressão logística multinomial para a hipertensão arterial a partir da classificação dos valores de PAS e PAD. 
Tabela 4. Razão de chances para desenvolvimento da pré-hipertensão e hipertensão arterial

\begin{tabular}{|c|c|c|c|c|}
\hline \multirow{2}{*}{ Variáveis } & Pré-hipertensivo & \multirow{2}{*}{ p-valor } & Hipertensivo & \multirow{2}{*}{ p-valor } \\
\hline & RC (IC95\%) & & RC (IC95\%) & \\
\hline \multicolumn{5}{|l|}{ Sexo } \\
\hline Feminino & 1 & & 1 & \\
\hline Masculino & $2,15^{\star \star}(1,65-2,81)$ & 0,001 & $2,38^{\star *}(1,90-2,98)$ & 0,001 \\
\hline \multicolumn{5}{|l|}{ Idade } \\
\hline & $1,03^{\star \star}(1,01-1,04)$ & 0,001 & $1,03^{\star *}(1,02-1,04)$ & 0,001 \\
\hline \multicolumn{5}{|l|}{ IMC } \\
\hline Normal & 1 & & 1 & \\
\hline Sobrepeso & $1,93^{\star \star}(1,36-2,73)$ & 0,001 & $1,71^{\star \star}(1,26-2,33)$ & 0,001 \\
\hline Obeso & $1,89^{\star *}(1,16-3,07)$ & 0,01 & $3,55^{\star *}(2,44-5,15)$ & 0,001 \\
\hline \multicolumn{5}{|l|}{ PC } \\
\hline Normal & 1 & & 1 & \\
\hline Elevado & $1,24(0,85-1,82)$ & 0,25 & $1,16(0,85-1,59)$ & 0,33 \\
\hline \multicolumn{5}{|l|}{ RCE } \\
\hline Normal & 1 & & 1 & \\
\hline Elevado & $0,81(0,54-1,22)$ & 0,32 & $1,38(0,97-1,96)$ & 0,06 \\
\hline
\end{tabular}

RC: razão de chance; IC: intervalo de confiança; IMC: índice de massa corporal; PC: perímetro da cintura; RCE: razão cintura estatura. *^ Critério de associação e de permanência no modelo = $p<0,05$.

Os resultados da associação entre hipertensão se deram para com o sexo, a idade e o IMC. Os homens têm 2,15 e 2,38 vezes mais chance de desenvolver pré-hipertensão e hipertensão respectivamente do que as mulheres. Quanto à idade, foi verificada uma chance de 1,03 vez mais a cada ano de desenvolver hipertensão arterial a partir de indicativos antropométricos considerados acima do recomendado. Quanto ao IMC, o grupo com sobrepeso e obesidade apresenta chances entre uma vez e meia e três vezes e meia de ter tanto pré-hipertensão quanto hipertensão quando comparado aos sujeitos normais (eutróficos).

\section{DISCUSSÃO}

Com base nos resultados, nas três análises realizadas, pode ser visto que as variáveis que estão associadas com a hipertensão são o sexo, a idade e o IMC, sendo semelhantes às encontradas em pesquisas prévias (10-13). Esses indicadores associados à hipertensão devem ser levados em conta na hora de realizar uma análise na verificação de possibilidade de um indivíduo desenvolver hipertensão. Os indicadores que estavam sendo utilizados em alguns centros de saúde como a RCE e o PC não estão fortemente associados com a hipertensão (12). Entretanto, somente para a classificação segundo a PAD, o PC entrou no modelo, mas foi o que teve a menor associação entre os indicadores antropométricos selecionados.

A prevalência de hipertensão ficou em torno de $8 \%$ a $14 \%$, porém abaixo do esperado para a população brasileira, que é de $20 \%$ a $40 \%(5,14)$. Logo, na presente pesquisa, embora sinalize quais os fatores que podem ser utilizados no diagnóstico da hipertensão arterial, certas limitações são levadas em consideração na anotação dos resultados descritos, sendo o caráter particular da amostra de indivíduos utilizados no estudo, ou seja, cidadãos do município de Curitiba-PR, que, por sua vez, limita a extrapolação dos descritos observados, e também o delineamento transversal do estudo não garante a precedência temporal das variáveis sobre a ocorrência da hipertensão arterial.

A literatura aponta que, com o avanço da idade, a pressão tende a aumentar, principalmente a sistólica, mas, para sujeitos que estão acima do peso e do sexo masculino, esse avanço pode ser mais acentuado (15). Outro estudo semelhante ao nosso, descrito por Wilsgaard e cols. (16), demonstra o aumento da pressão arterial sistólica e diastólica com o passar dos anos, verificando mudanças da pressão arterial de uma vez e meia para ambos os sexos.

Especificamente a partir do sexto decênio de vida, outros fatores de risco também são influenciadores da evolução da hipertensão arterial, como nível socioeco- 
nômico mais baixo; dieta inadequada (exemplo: excesso de sal); consumo elevado de bebida alcoólica; estilo de vida sedentário e concomitantemente, por meio desse, o excesso de peso (15-17). Mas destacamos não ser objetivo do estudo, e sim uma limitação deste, que foi o não controle da dieta, consumo de álcool, estilo de vida e situação socioeconômica. Mas, segundo recomendações da Sociedade Americana, Europeia e Canadense de Cardiologia (18-20), o estilo de vida pode ser um dos grandes fatores a ser modificado mediante práticas de atividades físicas aeróbias e resistidas $(30 \mathrm{a}$ 60 minutos, cinco a sete vezes na semana, com moderada intensidade), dieta, consumo de cigarros e bebida alcoólica e que se mostram influentes na prevenção das doenças cardiovasculares, principalmente a hipertensão arterial. Outro fator importante e limitante do estudo é quanto ao diagnóstico de hipertensão arterial, medida essa baseada em única mensuração, uma vez que o ideal seria realizar a média de duas medidas de pressão arterial obtidas em dois momentos distintos (15).

Demais variáveis ainda poderiam ser avaliadas e controladas para melhor inferência dos resultados, sendo que determinadas variáveis de confundimento tornam-se relevantes nas estimativas da associação entre o IMC e a hipertensão, assim como controle de medicamentos, momentos de estresse, alimentação inadequada, entre outros, podem interferir no diagnóstico, conforme apontado por outros estudos $(21,22)$.

A Sociedade Brasileira de Hipertensão (SBH) (5) alega que o aumento do peso corporal pode ser considerado um fator predisponente para o desenvolvimento da hipertensão arterial, sendo responsável por $20 \%$ a $30 \%$ dos casos da pressão arterial elevada. Setenta e cinco por cento dos homens e $65 \%$ das mulheres apresentam hipertensão diretamente atribuível ao excesso de peso.

No presente estudo ainda podemos observar que a obesidade foi a razão de chances mais elevada (OR $=3,6$ a 4,15) entre os fatores analisados, sendo que os modelos desenvolvidos calculam a probabilidade de cada indivíduo desenvolver hipertensão arterial diastólica ou sistólica partindo de variáveis antropométricas, mas, até o presente momento, não foram encontrados estudos com esse delineamento. Contudo, a Organização Mundial da Saúde (OMS), citada pela SBH (5), vem pondo em destaque que os pontos de corte para o sobrepeso $\left(25,0\right.$ e $\left.30,0 \mathrm{~kg} / \mathrm{m}^{2}\right)$ estão fortemente associados com risco de morbidade por meio das doenças cardiovasculares.

Assim como para a obesidade, Greenlund e cols. (2) demonstraram razão de chances do desenvolvimento de hipertensão arterial de OR: 3,34 e OR: 4,42, para mulheres e homens, respectivamente. Quando destacado o termo pré-hipertensão, o mesmo estudo descreve uma associação de OR: 1,46 e OR: 2,26, uma vez que, embora não classificando por faixa etária, esses resultados corroboram com os do nosso estudo.

Os resultados aqui apresentados podem ser utilizados no delineamento e na incorporação de políticas de saúde pública direcionadas à prevenção e ao controle dos fatores associados ao aparecimento da hipertensão. Assim, os trabalhos de promoção e prevenção de hipertensão deveriam ser focados para o controle com o avanço da idade, principalmente em indivíduos obesos e do sexo masculino. Dessa forma, a triagem poderia ser criada a partir de pontos de corte com esses indicadores analisando a probabilidade do desenvolvimento da hipertensão e seu avanço com o programa preventivo realizado.

Em contrapartida, os pontos fortes do estudo também são destacados, sendo: a diversidade demográfica e socioeconômica da amostra; a obtenção de medidas antropométricas e da pressão arterial por avaliação direta e não autorreferida, e o envolvimento de equipe multiprofissional no desenvolvimento do estudo, como realizado em estudos internacionais (23-25).

Haja vista os resultados encontrados estão em conformidade com a literatura (1-4,10-13,15-18,26-28), em que se ressalta que meios de prevenção para com o aumento de pessoas com excesso de peso em determinadas regiões do Brasil poderão apresentar as mesmas características para com o aumento da ocorrência de casos de hipertensão arterial.

Agradecimentos: agradecemos à Secretaria de Esporte e Lazer e Paraná Esporte por ceder os dados e ter colaborado para o desenvolvimento do estudo.

Declaração: os autores declaram não haver conflitos de interesse científico neste estudo.

\section{REFERÊNCIAS}

1. Chobanian AV, Bakris GL, Black HR, Cushman WC, Green LA, Izzo $\mathrm{JL} \mathrm{Jr}$; National Heart, Lung, and Blood Institute Joint National Committee on Prevention, Detection, Evaluation, and Treatment of High Blood Pressure; National High Blood Pressure Education Program Coordinating Committee. The Seventh Report of the Joint National Committee on Prevention, Detection, Evaluation, and Treatment of High Blood Pressure: the JNC 7 report. JAMA. 2003;289(19):2560-72.

2. Greenlund KJ, Croft JB, Mensah GA. Prevalence of heart disease and stroke risk factors in persons with prehypertension in the United States, 1999-2000. Arch Inter Med. 2004;164:2113-8. 
3. Polanczik CA. Decisão clínica em hipertensão arterial sistêmica baseada em análises econômicas. Rev Bras Hipertens. 2002;9:29-31.

4. Schocken DD, Benjamin EJ, Fonarow GC, Krumholz HM, Levy D, Mensah GA, et al. Prevention of Heart Failure: A Scientific Statement From the American Heart Association Councils on Epidemiology and Prevention, Clinical Cardiology Cardiovascular Nursing, and High Blood Pressure Research, Quality of Care and Outcomes Research Interdisciplinary Working Group, and Functional Genomics and Translational Biology Interdisciplinary Working Group. Circulation. 2008;117:2544-65.

5. Sociedade Brasileira de Cardiologia/Sociedade Brasileira de Hipertensão/Sociedade Brasileira de Nefrologia. VI Diretrizes Brasileiras de Hipertensão. Arq Bras Cardiol. 2010;95(1 supl.1):1-51.

6. Chirinos JA, Franklin SS, Townsend RR, Raij L. Body mass index and hypertension hemodynamic subtypes in the adult US population. Arch Intern Med. 2009;169(6):580-6.

7. Lohman TG, Roche AF, Martorell R. Anthropometric standardization reference manual. Champaign: Human Kinetics, 1988.

8. World Health Organization [WHO]. Consultation on Obesity. Obesity: preventing and managing the global epidemic Geneva, Switzerland: World Health Organization, 2000.

9. Tritschler K. Medida e avaliação em educação física e esportes de Barrow \& McGee. 5.ed. Barueri (SP): Manole, 2003.

10. Onis M, Habicht JP. Anthropometric reference data for international use: recommendations from a World Health Organization Expert Committee. Am J Clin Nutr. 1996;64:650-8.

11. Barbosa JB, Silva AAM, Santos AM, Monteiro FC, Barbosa MM, Figueiredo Neto JA, et al. Prevalência da hipertensão arterial em adultos e fatores associados em São Luís, MA. Arq Bras Cardiol. 2008;91(4):260-6.

12. Costa JS, Barcellos FC, Sclowitz ML. Prevalência de hipertensão arterial em adultos e fatores associados: um estudo de base populacional urbana em Pelotas, Rio Grande do Sul, Brasil. Arq Bras Cardiol. 2007;88(1):59-65.

13. Rosário TM, Scala LCN, França GVA, Pereira MR, Jardim PCBV. Fatores associados à hipertensão arterial sistêmica em Nobres, MT. Rev Bras Epidem. 2009;12(2):248-57.

14. Péresa DS, Magnaa JM, Vianab LA. Portador de hipertensão arterial: atitudes, crenças, percepções, pensamentos e práticas. Rev Saude Publica. 2003;37(5):635-42.

15. Chobanian AV, Bakris GL, Black HR, Cushman WC, Green LA. Seventh Report of the Joint National Committee on Prevention, Detection, Evaluation, and Treatment of High Blood Pressure. Hypertension. 2003;42(6):1206-52.

16. Wilsgaard T, Schirmer $H$, Arnesen E. Impact of body weight on blood pressure with a focus on sex differences the Tromso Study, 1986-1995. Arch Inter Med. 2000;160:2847-53.
17. Flegal KM, Carroll MD, Ogden CL, Johnson CL. Prevalence and trends in obesity among US adults, 1999-2000. JAMA. 2002;288:1723-7.

18. Hackam DG, Khan NA, Hemmelgarn BR, Rabkin SW, Touyz RM, Campbell NR, et al. The 2010 Canadian Hypertension Education Program recommendations for the management of hypertension: part 2 - therapy. Can J Cardiol. 2010;26(5):249-58.

19. Piepoli MF, Conraads V, Corra U, Dickstein K, Francis DP, Jaarsma $T$, et al. Exercise training in heart failure: from theory to practice. A consensus document of the Heart Failure Association and the European Association for Cardiovascular Prevention and Rehabilitation. Eur J Heart Failure. 2011;13:347-57.

20. Pollock ML, Franklin BA, Balady GJ, Chaitman BL, Fleg JL, Fletcher B, et al. AHA Science Advisory. Resistance exercise in individuals with and without cardiovascular disease: benefits, rationale, safety, and prescription: an advisory from the Committee on Exercise, Rehabilitation, and Prevention, Council on Clinical Cardiology, American Heart Association; position paper endorsed by the American College of Sports Medicine. Circulation. 2000;101:828-33.

21. Safar ME, Czernichow S, Blacher J. Obesity, arterial stiffness, and cardiovascular risk. J Am Society Nephrol. 2006;17(4):S109-11.

22. Schillaci G, Pirro M, Vaudo G, Mannarino MR, Savarese G, Pucci G, et al. Metabolic syndrome is associated with aortic stiffness in untreated essential hypertension. Hypertension. 2005;45(6):1078-82.

23. Deshmukh PR, Gupta SS, Dongre AR, Bharambe MS, Maliye $C$, Kaur $S$, et al. Relationship of anthropometric indicators with blood pressure levels in rural Wardha. Indian J Med Res. 2006;123: 657-64.

24. Ferreira I, Henry RA, Twisk JR, Van Mechelen W, Kemper HC, Stehouwer CD. Amsterdam Growth and Health Longitudinal Study. The metabolic syndrome, cardiopulmonary fitness, and subcutaneous trunk fat as independent determinants of arterial stiffness: the Amsterdam Growth and Health Longitudinal Study. Arch Inter Med. 2005;165(8):875-82.

25. Ogden CL, Carroll MD, Curtin LR, McDowell MA, Tabak CJ, Flegal KM. Prevalence of overweight and obesity in the United States, 1999-2004. JAMA. 2006;295:1549-55.

26. Lolio CA, Pereira JCR, Lotufo PA, Souza JMP. Hipertensão arterial e possíveis fatores de risco. Rev Saúde Publica. 1993;27(5):357-62.

27. Scuteri A, Najjar SS, Muller DC, Andres R, Hougaku H, Metter EJ, et al. Metabolic syndrome amplifies the age-associated increases in vascular thickness and stiffness. J Am Coll Cardiol. 2004;43(8):1388-95.

28. Vasan RS, Larson MG, Leip EP, Kannel WB, Levy D. Assessment of frequency of progression to hypertension in non-hypertensive participants in the Framingham Heart Study: a cohort study. Lancet. 2001;358:1682-6. 